\title{
Men are from Mars and women are from Venus: The nuclear cardiology point of view
}

\author{
Nicola Riccardo Pugliese, $M D{ }^{a}$ and Alessia Gimelli, $M D^{b}$ \\ a Department of Clinical and Experimental Medicine, University of Pisa, Pisa, Italy \\ b Fondazione Regione Toscana "Gabriele Monasterio", Pisa, Italy
}

Received Aug 29, 2019; accepted Aug 29, 2019

doi:10.1007/s12350-019-01891-w

\section{See related article, pp. 1569-1582}

Single-photon emission computed tomography (SPECT) has a pivotal role in the non-invasive evaluation of known or suspected coronary artery disease (CAD), thanks mainly to the myocardial perfusion imaging (MPI) assessment. ${ }^{1}$ The use of electrocardiographic gating also enabled accurate measurements of left ventricle (LV) wall motion, ejection fractions, and volumes, which allows the possibility to integrate several important functional information to perfusion evaluation. ${ }^{2}$ In the last years, the introduction of dedicated cardiac cameras equipped with Cadmium-Zinctelluride (CZT) technology has further improved the spatial and temporal resolution, with a significant reduction in the acquisition time and injected dose. ${ }^{3-5}$ Nevertheless, attention should also be paid to unexpected artifacts using CZT detectors too. For example, a supine position could limit the evaluation of the inferior wall because of attenuation artifacts from diaphragmatic elevation or adjacent abdominal visceral activity, although less than expected with standard SPECT. Briefly, soft tissue attenuation of tracer activity can result in artifactual perfusion abnormalities in the right coronary artery and left circumflex territories. In such cases, it is advisable to add prone or upright imaging, because in the new position artifactual defects shall resolve or change their location, whereas true perfusion defects shall persist. ${ }^{2}$ At present, there is more experience with the conventional supine/prone imaging, $4,6,7$

\footnotetext{
Reprint requests: Alessia Gimelli, MD, Fondazione Regione Toscana "Gabriele Monasterio", via Moruzzi n.1, 56124 Pisa, Italy; gimelli@ftgm.it

J Nucl Cardiol 2021;28:1583-5.

$1071-3581 / \$ 34.00$

Copyright (C) 2019 American Society of Nuclear Cardiology.
}

while data about upright cardiac images are limited, particularly in female patients. ${ }^{8,9}$ Upright imaging has some benefits as compared with a prone acquisition because the former does not require the patient to raise both hands during image collection, so body posture is stable, and body movements are reduced. Also, prone imaging affects respiration: abdominal breathing prevails over chest breathing at rest, but the prone position places pressure on the abdomen and promotes chest breathing. Therefore, the upright position makes breathing easier with the patient in a relaxed state. At the same time, the movements of the diaphragm are broader in abdominal breathing than in chest breathing; thus, both imaging methods improve evaluation of the inferior wall for breathing-related artifacts. A 2-position imaging approach can be particularly helpful in female patients, in which breast attenuation artifact adds to the issues outlined above. Breast attenuation artifact is challenging when the left breast position varies between the restand-stress images. It can be confirmed by repeating the acquisition with the left breast repositioned or with prone or upright imaging to reduce breast attenuation artifacts. $^{2}$ There are few data in the literature on the direct comparison between prone and upright imaging. Nakaya et al. observed a higher rate of suppression of soft tissue attenuation artifacts with prone imaging compared to upright artifacts using a conventional gamma camera, but the difference did not affect visual evaluation. ${ }^{10}$ Noteworthy, all the previously described artifacts were significantly more familiar with the traditional Anger cameras, which suffered from inaccurate $\mathrm{LV}$ reconstructions, above all in smaller hearts. ${ }^{11}$ On the contrary, CZT cameras allow the physician to rely on a single position (i.e., supine) in most cases, both in male and female patients. ${ }^{2,4,12}$ The higher reliability of CZT detectors also lies with the acquisition of a comprehensive dataset on LV function from gated SPECT that can be integrated with MPI into the final interpretation. Therefore, the physician can readily verify whenever 
fixed, non-reversible perfusion defects are associated with abnormalities in wall motion or myocardial systolic thickening; a mismatch is more likely to be an artifact, notably if the clinical data do not support a prior infarction. Finally, attenuation correction is also possible with an attached full-size computed tomography (CT), which is a useful method to improve the diagnostic accuracy of the inferior wall ischemia. ${ }^{13}$ Unfortunately, $\mathrm{CT}$ attenuation correction is not widely used due to the significant cost increase of the hardware. The assessment of the potential additional value of upright imaging to the conventional supine imaging is useful to weigh the need for a longer acquisition and manage discordant findings between upright and supine images. In the current issue of the Journal of Nuclear Cardiology, Muhammad W. Athar and associates analyzed supine and upright images after stress and at rest using a DSPECT CZT dedicated cardiac camera. ${ }^{14}$ The authors found that MPI assessment by multiple nuclear test quantitative variables was more accurate in supine than upright imaging, both in male and female patients. These findings, if confirmed by more extensive studies, may limit the patient selection for upright CZT imaging system.

The focus on female patients is of utmost importance, because the incidence and prevalence of cardiovascular (CV) disease, and mainly stable ischemic heart disease (SIHD), significantly increased in women compared to men in the last three decades. ${ }^{15}$ According to the current guidelines, patients with intermediate pretest SIHD risk should undergo non-invasive imaging, and SPECT-derived MPI with exercise or pharmacologic stress testing represents a robust approach for diagnosing flow-limiting CAD. ${ }^{1}$ Still, appropriate delineation and utilization of guideline-directed care for women with SIHD remain a vital goal. This could be related to the fact that women commonly present with more atypical, less exertional symptoms, which confound candidate selection and accurate assessment of pre-test risk. ${ }^{1}$ Women presenting with the suspect of SIHD consistently receive less intensive medical care and are under-referred to $\mathrm{CV}$ imaging procedures as well. ${ }^{16,17}$ Some technical reasons limited the conventional Anger cameras, as safety issues and low diagnostic accuracy. ${ }^{18,19}$ CZT-based cardiac imaging succeeded in reducing radiation exposure and improve CAD diagnosis as well, thanks to robust software programs for MPI and LV function assessment. Our group demonstrated that sex-based normal limits and software interpretation provide high diagnostic accuracy for detection of obstructive CAD without significant sex differences and irrespective of the number of the vessel with CAD or the stress protocol (exercise or pharmacologic stress test). ${ }^{4,5}$ In this respect, the results of Athar et al. confirm the need of different sex-based reference limits (a supine $\mathrm{SSS} \geq 3$ in men and $\mathrm{SSS} \geq 2$ in women) to obtain high sensitivity $(70.7 \%$ and $90 \%$ sensitivity in men and women, respectively), even if this is at the expense of a significantly lower specificity for women $(32.9 \%)$ as compared to men $(72.2 \%)$. The authors demonstrated that the introduction of a quantitative measure of defect reversibility increases diagnostic accuracy in female patients to $92.9 \%$ sensitivity and $54.3 \%$ specificity. ${ }^{14}$ Indeed, a correct description of MPI defects should include the defect size or extent, the severity of perfusion defect, the extent of reversibility, and the location (based on the 17-segment model and/or coronary artery territory). The best parameter to sum up all the previous characteristics is SSS,${ }^{1,2}$ which is able to identify both ischemia and scar, thus providing an independent value in CAD diagnosis and risk stratification. ${ }^{4,20}$ Therefore, SSS expresses perfusion defect size and extent, reflecting the presence of anatomic disease related to scar and/or ischemia. The identification of reversible perfusion defects represents a more specific marker of ischemia. Defect reversibility can be performed visually, but it is limited by the high inter-observer variability. A quantitative assessment is more appropriate, and the summed difference score (SDS) is the most accepted measure for detection of reversible perfusion defects. ${ }^{2}$ Athar et al. propose the use of total quantitative change in defect reversibility in the entire LV (QREV) in the supine position, defined as an increase in total LV defect per cent at stress compared to rest or as any 5\% or greater increase in reversibility from stress to rest. In the present study, SDS was not an independent predictor of CAD when tested in multivariable models. The authors speculated that these findings could be related to the specifically chosen endpoint, namely the presence of angiographic stenosis defined as at least one vessel with luminal diameter stenosis $>70 \%$ and not myocardial ischemia. According to this reasoning, SSS alone provides to the multivariable models all the info contained in the SDS, because SSS accounts for the anatomic disease that is related both to scar and ischemia. Instead, QREV has been successfully included in the model as a dichotomous variable with a priori definition, in contrast with the SSS cut-offs, which were identified by ad hoc ROC analysis.

The present study is a welcome addition to improve CAD diagnostic strategies in women. It has the merit of including a large population $(n=260$ consecutive patients) with a wide spectrum of cardiac diseases and no exclusion criteria, with a significant proportion of female patients. Their findings underline that diagnostic accuracy in females is still lower than in males using some models of CZT cameras. However, they 
demonstrated that the predictive ability to identify CAD in female patients could be significantly increased by the assessment of reversible perfusion defects. The most important limitation of the research is the retrospective and single-center nature of the study. Moreover, the authors pointed out a prevalent problem, that is the difficulty faced by women undergoing exercise stress to reach the target heart rate on the treadmill. ${ }^{4}$ The physician is always asked to choose the correct protocol to ensure the best performance result. It is conceivable that a pharmacologic stress test has a diagnostic accuracy higher than a submaximal exercise test, but until now, no study focused on this problem has been conducted. More extensive and prospective trials are needed to fill the blanks and confirm the findings of the present study.

\section{Disclosure}

The authors Nicola Riccardo Pugliese and Alessia Gimell declare no conflict of interests.

\section{References}

1. Task Force Members, Montalescot G, Sechtem U, et al. 2013 ESC guidelines on the management of stable coronary artery disease. Eur Heart J 2013;34:2949-3003.

2. Dorbala S, Ananthasubramaniam K, Armstrong IS, et al. Single photon emission computed tomography (SPECT) myocardial perfusion imaging guidelines: Instrumentation, acquisition, processing, and interpretation. J Nucl Cardiol 2018;25:1784-846.

3. Gimelli A, Pugliese NR, Kusch A, et al. Evaluation data about accuracy of cadmium-zinc-telluride imaging in detecting single and multivessel coronary artery disease: Focus on gender differences. Data Br 2018;21:1654-8.

4. Gimelli A, Pugliese NR, Kusch A, et al. Accuracy of cadmiumzinc-telluride imaging in detecting single and multivessel coronary artery disease: Is there any gender difference? Int $\mathrm{J}$ Cardiol 2019;274:388-93.

5. Gimelli A, Bottai M, Genovesi D, et al. High diagnostic accuracy of low-dose gated-SPECT with solid-state ultrafast detectors: Preliminary clinical results. Eur J Nucl Med Mol Imaging 2012;39:83-90.

6. Taasan V, Wokhlu A, Taasan MV, et al. Comparative accuracy of supine-only and combined supine-prone myocardial perfusion imaging in men. J Nucl Cardiol 2016;23:1470-6.

7. Nishina H, Slomka PJ, Abidov A, et al. Combined supine and prone quantitative myocardial perfusion SPECT: Method development and clinical validation in patients with no known coronary artery disease. J Nucl Med 2006;47:51-8.

8. Ben-Haim S, Almukhailed $\mathrm{O}$, Neill $\mathrm{J}$, et al. Clinical value of supine and upright myocardial perfusion imaging in obese patients using the D-SPECT camera. J Nucl Cardiol 2014;21:478-85.

9. Betancur J, Hu LH, Commandeur F, et al. Deep learning analysis of upright-supine high-efficiency SPECT myocardial perfusion imaging for prediction of obstructive coronary artery disease: A multicenter study. J Nucl Med 2019;60:664-70.

10. Nakaya K, Onoguchi M, Nishimura Y, et al. Comparison between prone and upright imaging of the inferior wall using $201 \mathrm{TlCl}$ myocardial perfusion SPECT. J Nucl Med Technol 2017;45:304-8.

11. Kane GC, Karon BL, Mahoney DW, et al. Progression of left ventricular diastolic dysfunction and risk of heart failure. JAMA 2011;306:856-63.

12. Nudi F, Iskandrian AE, Schillaci O, et al. Diagnostic accuracy of myocardial perfusion imaging with CZT technology: systemic review and meta-analysis of comparison with invasive coronary angiography. JACC Cardiovasc Imaging 2017;10:787-94.

13. Pazhenkottil AP, Ghadri JR, Nkoulou RN, et al. Improved outcome prediction by SPECT myocardial perfusion imaging after CT attenuation correction. J Nucl Med 2011;52:196-200.

14. Athar MW, Waqar F, Dwivedi AK, et al. Effects of gender and defect reversibility on detection of coronary disease with an upright and supine cadmium-zinc-telluride camera. J Nucl Cardiol (In press).

15. Ouyang P, Wenger NK, Taylor D, et al. Strategies and methods to study female-specific cardiovascular health and disease: A guide for clinical scientists. Biol Sex Differ 2016;7:19.

16. Hachamovitch R, Berman DS, Kiat H, et al. Effective risk stratification using exercise myocardial perfusion SPECT in women: Gender-related differences in prognostic nuclear testing. J Am Coll Cardiol 1996;28:34-44.

17. Taillefer R, DePuey EG, Udelson JE, et al. Comparative diagnostic accuracy of Tl-201 and Tc-99m sestamibi SPECT imaging (perfusion and ECG-gated SPECT) in detecting coronary artery disease in women. J Am Coll Cardiol 1997;29:69-77.

18. Verberne HJ, Acampa W, Anagnostopoulos C, et al. EANM procedural guidelines for radionuclide myocardial perfusion imaging with SPECT and SPECT/CT: 2015 revision. Eur J Nucl Med Mol Imaging 2015;42:1929-40.

19. Montalescot G, Sechtem U, Achenbach S, et al. 2013 ESC guidelines on the management of stable coronary artery disease: The task force on the management of stable coronary artery disease of the European Society of Cardiology. Eur Heart J 2013;34:2949-3003.

20. Iskander S, Iskandrian AE. Risk assessment using single-photon emission computed tomographic technetium- $99 \mathrm{~m}$ sestamibi imaging. J Am Coll Cardiol 1998;32:57-62.

Publisher's Note Springer Nature remains neutral with regard to jurisdictional claims in published maps and institutional affiliations. 